\title{
Optimization of the electrical connection topology of a tidal farm network
}

\author{
Eyman Fakhri ${ }^{1}, *$, Salvy Bourguet ${ }^{2}$, Jérôme Thiébot ${ }^{1}$, Mohamed Machmoum ${ }^{2}$ and Hamid Gualous ${ }^{1}$ \\ ${ }^{1}$ Laboratoire Universitaire des Sciences Appliquées de Cherbourg (LUSAC), Normandie Univ, UNICAEN, 14000 Caen France. \\ ${ }^{2}$ Institut de Recherche en Énergie Électrique de Nantes Atlantique (IREENA), Université de Nantes, 44602 Saint Nazaire, France.
}

\begin{abstract}
This article presents an approach to optimize the electrical connection topology of tidal energy converters in a tidal farm. The methodology is based on a genetic algorithm (GA). The main purpose is to present a technique of coding to find the best electrical connection topology of the tidal farm network. The optimization model takes into account the energy loss in the submarine cables. The model gives as its output the optimal number of turbine clusters connected to each offshore substation, the number of turbines in each cluster, the cross-section of MV and HV cables, the connection design for each cluster of turbines as well as the number and the locations of the offshore substations. A particle swarm optimization algorithm (PSO) is used to confirm the results obtained with the GA. The optimization approach is applied to the Fromveur Strait (France).
\end{abstract}

\section{Introduction}

Both industrialized and developing countries have to meet growing energy needs. In the context of climate emergency, several countries have shown a strong interest to reduce greenhouse gases and increase significantly the share of renewable energies in their national energy production. To meet this objective, marine renewable energies can contribute substantially to new energy sources that do not emit greenhouse gases. Compared to the other marine energy sources, the tidal energy extracted using Tidal Energy Converters (TECs) has the great advantage of being highly predictable, facilitating the management of its production and distribution [1]. Nevertheless, the extraction of this energy requires significant investment. Indeed, one of the major challenges facing the development of tidal turbines is the cost of producing energy, which is nowadays significantly higher than that produced from fossil fuels. It is therefore now necessary to develop tools to optimize the entire production chain and to reduce the cost of the tidal energy production.

The energy cost of a TEC farm depends on the configuration of the power grid. In marine renewable energy applications, the electrical system cost represents between 15 and $30 \%$ of the total investment cost, of which $5 \%$ are the costs of submarine cables $[2,3]$. The cost of the electrical system is influenced by several factors which are intertwined [3]. These factors such as the energy loss and the investment cost of the Electrical Connection Topology (ECT) have to be studied and optimized beforehand. The implementation of an optimization procedure during the project planning stage intends to reach the best compromise between technical performance and investment cost [4].

For large tidal farms, the energy produced by TECs must be collected at one or more Offshore Sub-Stations (OSSs) and transported by submarine cables to the Onshore Connection Point (OCP) (Fig. 1). Several optimization approaches have been proposed in the literature to optimize the ECT. These approaches have mainly focused on offshore wind farm applications where the technological maturity level is higher than that of tidal farms. Metaheuristic optimization methods have proven their effectiveness and are widely used in the literature. For instance, Hou et al. [5] focussed mainly on cable optimization using simple algorithms, such as the minimum spanning tree algorithm or a dynamic minimum spanning tree as in [6]. Others adopted broader optimization approaches including the number and the location of OSSs, the ECT as well as the selection of electrical components in terms of voltage level. To this end, González et al. [7] used a particle swarm optimization (PSO) algorithm whereas Dahmani et al. [8] developed an optimization method based on a mixed minimum spanning tree and a Genetic Algorithm (GA). Only a few studies have focused on the optimization of the ECT of tidal farms. The only existing studies take into consideration a limited number of optimization variables such as the length of submarine cables and their cross-sections $[9,10]$. In this work, we present an optimization model based on a GA which takes into account a large panel of optimization variables. This optimization model takes into account the energy loss in the submarine cables. The model gives as its output the internal design of the connection topology among the TECs and the electrical Offshore SubStation (OSS), the

\footnotetext{
* Corresponding author: eyman.fakhri@unicaen.fr
} 
cross-section of MV and HV cables, and the number and locations of OSSs as well the sizing of its components. A PSO algorithm will be used to confirm the GA results.

The optimization model will be applied on a hypothetical hydrodynamic site containing 45 TECs, located in the Fromveur Strait (France). This tidal turbine site represents the second greatest tidal potential in France.

The mathematical formulation of the optimization problem is provided in detail in section II. In section III, the optimization methodology is presented. The results are discussed in Section IV.

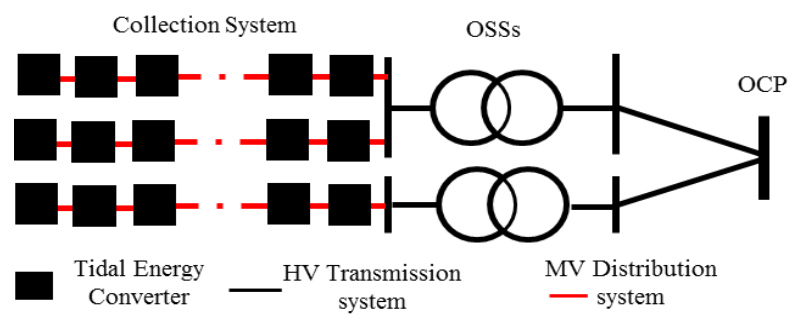

Fig.1. General ECT configuration of a tidal farm

\section{Mathematical formulation of the Optimization problem}

The ECT optimization problem consists in finding the optimal number of turbine clusters, the number of turbines and connection design for each cluster, the cross-section of MV and HV cables as well as the number and the locations of the OSSs. The optimization approach takes also into account the energy loss as well as the amount of reactive power required to compensate. The objective function of the electrical connection topology $\mathrm{F}_{\mathrm{ETC}}$ is a multicriteria function, defined as the sum of the investment cost $\mathrm{C}_{\text {invest }}$ and the cost of the energy loss in the connection network $\mathrm{C}_{\text {losses }}$.

$$
F_{E C T}=\min \left(C_{\text {losses }}+C_{\text {invest }}\right)
$$

\subsection{Cost of the energy loss}

A load flow calculation is adopted in this work to evaluate the power losses $\mathrm{P}_{\text {losses. }}$ To feed the load flow algorithm, line and bus data are constructed from the connection matrix (A) and from the submarines cables data used (the construction of $\mathrm{A}$ is detailed in the following section). The TECs are modeled as PQ-nodes. The algorithm provides the modules and phases voltages ( $\mathrm{V}$ and $\alpha$ ) at different nodes in the grid. The load flow calculation consists of solving both (2) and (3) using the universal method of Newton-Raphson [11].

$$
\begin{aligned}
& P_{i, j}=V^{2}{ }_{i}\left(g_{i, j}-G_{i, j}\right)+V_{i} V_{j}\left[G_{i, j} \cos \left(\alpha_{i^{-}} \alpha_{j}\right)+B_{i, j} \sin \left(\alpha_{i^{-}} \alpha_{j}\right)\right] \\
& Q_{i, j}=V^{2}{ }_{i}\left(h_{i, j}-B_{i, j}\right)+V_{i} V_{j}\left[G_{i, j} \sin \left(\alpha_{i^{-}} \alpha_{j}\right)-B_{i, j} \cos \left(\alpha_{i^{-}} \alpha_{j}\right)\right]
\end{aligned}
$$

Where $\mathrm{P}_{\mathrm{i}, \mathrm{j}}$ and $\mathrm{Q}_{\mathrm{i}, \mathrm{j}}$ are respectively the active and reactive power transmitted from the node " $\mathrm{i}$ " to node “ $\mathrm{j}$ ", $\mathrm{y}_{\mathrm{i}, \mathrm{j}}=\mathrm{g}_{\mathrm{i}, \mathrm{j}}+\mathrm{h}_{\mathrm{i}, \mathrm{j}}$ is the parallel admittance in a $\pi$ diagram and $\mathrm{Y}_{\mathrm{i}, \mathrm{j}}=\mathrm{G}_{\mathrm{i}, \mathrm{j}}+\mathrm{B}_{\mathrm{i}, \mathrm{j}}$ is the global admittance of the network.
Assuming that some profit PR will be realized, the cost induced by these power losses is calculated as follows:

$$
\mathrm{C}_{\text {losses }}=\left(\mathrm{P}_{\text {losses }} \cdot \mathrm{T}_{\mathrm{h}} \cdot \mathrm{C}_{\text {elect }}\right) /(1-\mathrm{PR})
$$

Where $C_{\text {elect }}$ is the electricity cost, which is estimated in our study at $0.145 € / \mathrm{kWh}$ and $T_{h}$ is the number of the tidal farm operating hours.

\subsection{Electrical investment cost}

It is assumed that the total ECT investment is made in the first year and paid during the lifetime $\mathrm{T}$ of the tidal farm with a bank interest rate $r$. $C_{\text {invest }}$ is therefore calculated as follows:

$$
\left.C_{\text {invest }}=\left\{C_{0 . r .}(1+r)^{T}\right\} /\left\{\left[(1+r)^{T}-1\right)\right] \cdot[1-P R]\right\}
$$

Where $\mathrm{C}_{0}$ is the initial investment cost.

The components of the electricity network that are considered in the initial investment cost are: the cost of $\mathrm{MV}$ and $\mathrm{HV}$ cables $\mathrm{C}_{\text {cable, the cost of reactive energy }}$ compensation equipments $\mathrm{C}_{\mathrm{ec}}$ and the cost of the OSSs $\mathrm{C}_{\text {Oss }}$. The initial investment cost can be defined as the sum of the three costs and calculated as follows:

$$
C_{0}=C_{\text {cable }}+C_{\text {OSS }}+C_{e c}
$$

The cable cost is calculated as follows [12]:

$$
C_{\text {cable }, i}=A_{c}+B_{c}+\exp \left[\left(S_{n, i} . C_{c}\right) / 10^{2}\right]
$$

Where $A_{c}, B_{c}$ and $C_{c}$ are the coefficients of the submarine cable cost which depend on the voltage level and are estimated in [12] as bellow:

Table 1. Coefficient values of the submarine cable cost model.

\begin{tabular}{|c|c|c|c|}
\hline $\begin{array}{c}\text { Voltage } \\
\text { level }\end{array}$ & $A_{c}$ & $B_{c}$ & $C_{c}$ \\
\hline $22 \mathrm{kV}$ & 0.0217 & 0.0921 & 5.3671 \\
\hline $132 \mathrm{kV}$ & 0.2011 & 0.0234 & 1.5505 \\
\hline
\end{tabular}

Reactive energy compensation equipment is installed at the ends of the HVAC cables. $\mathrm{C}_{\mathrm{ec}}$ is proportional to the amount of total reactive power $\mathrm{Q}_{\text {comp }}$ to be compensated and is calculated as follows [12]:

$$
C_{e c}=-93015.6+165 . Q_{\text {comp }}{ }^{0.4473}
$$

An OSS includes a step-up transformer and its associated switchgear [13]. The cost of the OSS platform is estimated by the model proposed in [14]. The cost of an OSS is defined as the sum of the costs of the step-up transformer calculated by (9) [14] [13]; the switchgear is calculated with (10)[12], and the cost of the offshore platform is calculated with (11) [15].

$$
\begin{aligned}
& C_{t r}=-157727+280.5 \mathrm{~S}_{\mathrm{tn}}^{0.4473} \\
& C_{S G}=30400+0.57 \mathrm{U}_{\text {rated }} \\
& C_{p l}=2+0.07 \mathrm{~S}_{\mathrm{tn}}
\end{aligned}
$$

Where Stn is the rated power of the transformer installed in the OSS and $U_{\text {rated }}$ is the rated voltage of the switchgear, line to line (V). 


\section{Optimization methodology}

\subsection{Connection matrix construction}

The ECT is modeled by a connection matrix A as shown in Fig.2 and as adopted in [8] (in the offshore wind turbine context). The elements of this matrix are either 1 if the bus of line $i$ is connected to the bus of column $j$ or 0 if there is no connection. The connection matrix shown in Fig. 2 is a square, symmetric matrix of size $n_{A}$.

$$
n_{A}=N_{T E C}+n_{O S S}+1
$$

Where $\mathrm{n}_{\mathrm{OSS}}$ is the number of OSSs and $N_{T E C}$ is the number of TECs in the farm.

The connection matrix is composed of 3 submatrices: namely $\mathrm{A}_{\text {TEC-TEC, }} \mathrm{A}_{\text {TEC-OSS }}$ and $\mathrm{A}_{\text {TEC-OCP. }}$ They represent respectively the connection topology between the TECs, the connection topology between OSS(s) and TECs and the connection topology between $\mathrm{OSS}(\mathrm{s})$ and the OCP.

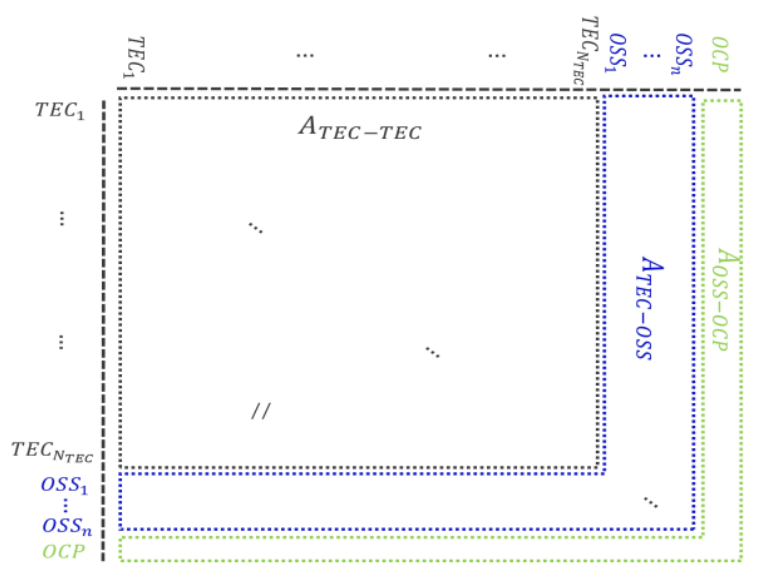

Fig. 2. Schematic representation of the connection matrix.

\subsection{Optimization Algorithms}

The GA permits to solve both constrained and unconstrained optimization problems. This method is inspired by Charles Darwin's theory of natural evolution. The basic principle of the GA is the evolution during the simulation time of a population. The fittest individuals are selected for reproduction to produce offspring of the next generation. Three operators called "selection", "crossover" and "mutation" are applied to generate new individuals. Each individual is encoded into a chromosome $\mathrm{X}_{\mathrm{GA}}$. The gens of the chromosome are binary values.

The PSO is a population based stochastic optimization technique developed by Eberhart and Kennedy in 1995. It is inspired by social behavior of bird flocking or fish schooling [16]. The speed and position of each particle is updated according to the following equation [17]:

$\mathrm{V}_{\mathrm{t}+1}=\omega \mathrm{V}_{\mathrm{t}}+\mathrm{r}_{1} \mathrm{c}_{1}\left(\mathrm{p}_{\mathrm{i}, \mathrm{t}}-\mathrm{x}_{\mathrm{t}}\right)+\mathrm{r}_{2} \mathrm{c}_{2}\left(\mathrm{p}_{\mathrm{g}, \mathrm{t}}-\mathrm{x}_{\mathrm{t}}\right)$

$\mathrm{X}_{\mathrm{t}+1}=\mathrm{x}_{\mathrm{t}}+\mathrm{v}_{\mathrm{t}+1}$

Where $c_{1}$ and $c_{2}$ are two positive constants, $r_{1}$ and $r_{2}$ are two random functions in the range [0-1], $v_{t}$ is the velocity at the time $t, x_{t}$ is the position of the particle, $p_{i, t}$ is the best previous position, $\mathrm{p}_{\mathrm{g}, \mathrm{t}}$ is the est candidate solution for the entire swarm, and $\omega$ is the inertia weight coefficient.

Each chromosome generated by the GA or the PSO algorithm represents a different topology of connection. The general chromosome structure of $\mathrm{X}_{\mathrm{GA}}$ is presented in Fig.3. It is composed of 4 sub-chromosomes:

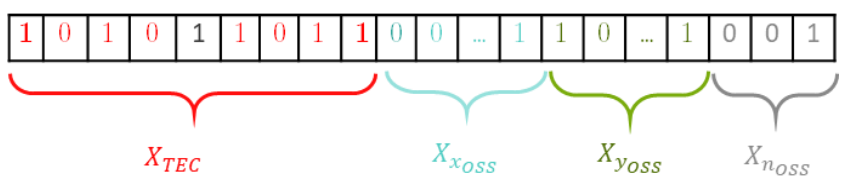

Fig. 3. General structure of $\mathrm{X}_{\mathrm{GA}}$.

1. The sub-chromosome $X_{\text {TEC }}$ of size $N_{V, T E C}$ represents the connection topology between the TECs and is used to fill the $A_{\text {TEC-TEC }}$ matrix. $\mathrm{N}_{\mathrm{V} \text {,TEC }}$ depends on the number of TECs and is calculated as follows:

$$
N_{V, T E C}=\left[N_{T E C}\left(N_{T E C}-1\right)\right] / 2
$$

2. The sub-chromosome $X_{n, \text { oss }}$ represents the number of OSSs. This number determines the size of A. In our case, we set the maximum number of OSSs to 3 . Thus $\mathrm{X}_{\mathrm{n}, \mathrm{OSS}}$ is encoded in 3 bits $\left(\mathrm{N}_{\mathrm{vn}, \mathrm{OSS}}=2\right)$.

3. The sub-chromosomes $\mathrm{X}_{\mathrm{x}, \mathrm{OSS}}$ and $\mathrm{X}_{\mathrm{y}, \mathrm{OsS}}$ represent the positions of the OSSs the $\mathrm{x}$ and the $\mathrm{y}$ - axis, respectively. It is coded as a 4 bit binary string:

$$
N_{V, x O S S}=N_{V, y O S S}=4 n_{O S S}
$$

The number of the variables of $\mathrm{X}_{\mathrm{GA}}$ (i.e. $\mathrm{N}_{\mathrm{v}}$ ) is then calculated as follows:

$$
N_{V}=N_{V, T E C}+N_{V, x O S S}+N_{V, y O S S}+\mathrm{N}_{\mathrm{vn}, \mathrm{OSS}}
$$

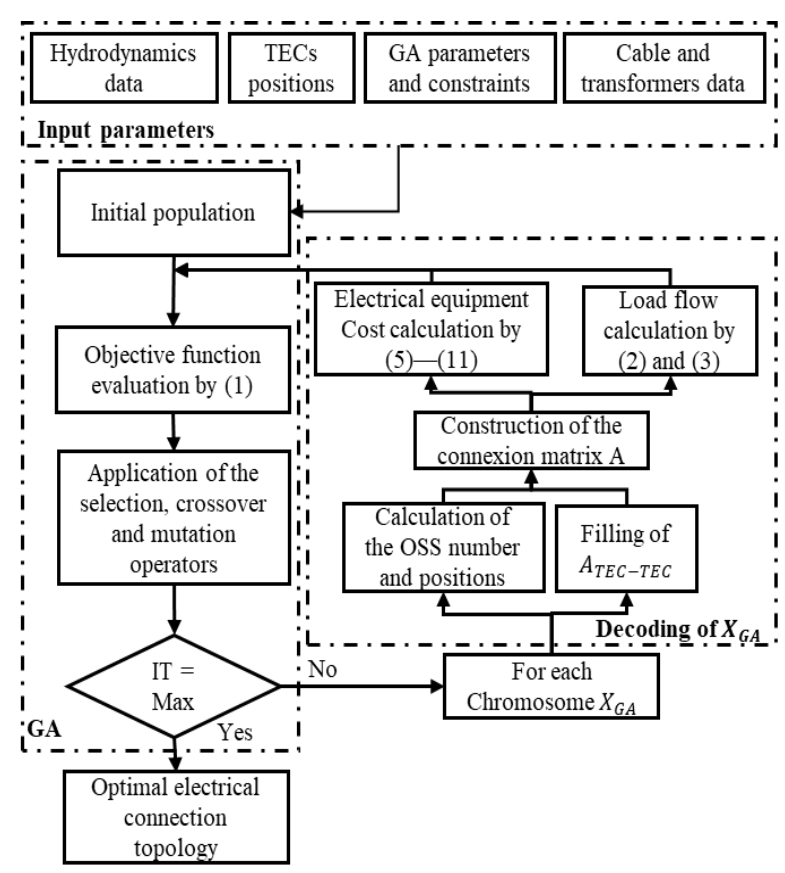

Fig. 4. Overview of the optimization method.

To minimize the search space, we reduce the number of variables by adopting the two simplifications used in [8]. (i) Each TEC can only be connected to its eight direct 
neighbours. (ii) The OSS is connected to the closest TEC from each cluster. These simplifications are sensible considering that the connection between two remote nodes is not optimal. The overall optimization procedure is represented in Fig. 4.

\section{Results and discussion}

The turbine characteristics are those of the Atlantis AR1500 tidal turbine. The turbine used in this application is a horizontal axis turbine. The rotor diameter of the turbine is $18 \mathrm{~m}$. The cut-in, rated and cut-out speed of the turbine are respectively $0.5 \mathrm{~m} / \mathrm{s}, 3$ $\mathrm{m} / \mathrm{s}$ and $5 \mathrm{~m} / \mathrm{s}$. The optimization approach is applied to a $900 \mathrm{~m} \times 900 \mathrm{~m}$ area located in the Fromveur Strait. The farm is composed of 45 TECs arranged as illustrated in Fig 5. The voltage levels of the network are $22 \mathrm{kV}$ for the collection system and $132 \mathrm{kV}$ for the transmission system. AC transmission is ensured by a single threecore XLPE subsea cable. The sizes of submarine cables $\mathrm{C}_{\text {,size }}$ and the power of transformers $\mathrm{T}_{\text {,size }}$ are standardized. $\mathrm{C}_{\mathrm{MV} \text {,size }}\left(22 \mathrm{kV}, \mathrm{mm}^{2}\right)=[35,50,70,95,120$, $150,815,240], \mathrm{C}_{\mathrm{HV} \text {,size }}\left(132 \mathrm{kV}, \mathrm{mm}^{2}\right)=[185,240,300$, $400,500,630,800,1000], \mathrm{T}_{\text {size }}(\mathrm{MVA})=[10,25,40,50$, $100,125,150,180,200]$. The OCP considered in this work is located approximately $20 \mathrm{~km}$ away from the center of the farm. The parameters of the GA and PSO are synthesized in Table 2 .

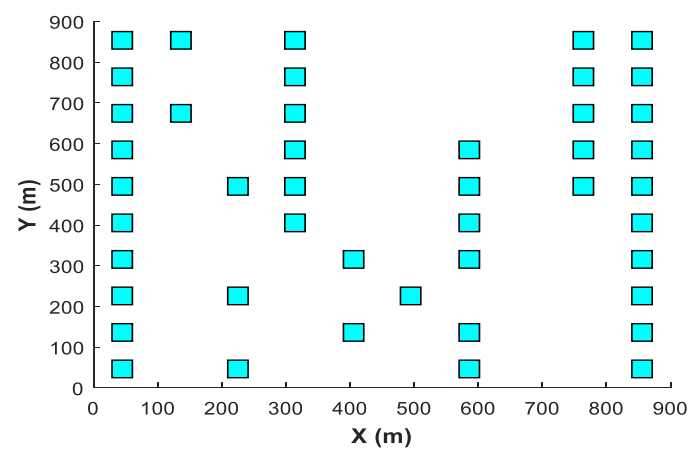

Fig. 5. TECs positions.

Table 2. GA and PSO parameters.

\begin{tabular}{|c|c|c|}
\hline & Quantity & Value \\
\hline \multirow{6}{*}{ 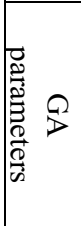 } & Number of variables & 142 \\
\hline & Population Size & 50 \\
\hline & Max. number of iterations & 3000 \\
\hline & Crossover percentage & 0,8 \\
\hline & Mutation rate & 0,02 \\
\hline & Number of mutants & random \\
\hline \multirow{8}{*}{ 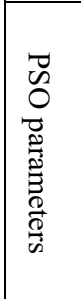 } & Number of variables & 142 \\
\hline & Swarm size & 50 \\
\hline & Max. number of iterations & 3000 \\
\hline & Initial ; Final cognitive parameter & $2.5 ; 0.5$ \\
\hline & Initial ; Final social parameter & $0.5 ; 2.5$ \\
\hline & Constriction factor & 1 \\
\hline & Inertia weight coefficient & 1 \\
\hline & Initial cognitive parameter & 2.5 \\
\hline
\end{tabular}

The optimal ECTs using a GA and a PSO algorithm are represented in Fig.6. The two electrical topologies are very similar, especially in terms of the number of turbine clusters connected to each OSS, the number of turbines in each cluster, the cross-section of MV and HV cables and the number and locations of OSSs. Indeed, for both connection topologies, the connections between the TECs and between the TECs and the OSS are represented respectively by the $\mathrm{A}_{\mathrm{TEC}-\mathrm{TEC}}$ and $\mathrm{A}_{\mathrm{TEC}-\mathrm{OSS}}$ submatrices. These connections are realized without any crossing between cables. The optimal number of OSS is 1 in a central positioning along $\mathrm{x}$-axis $((\mathrm{x}, \mathrm{y})=(495,315))$ with a transformer of 100 MVA. The TECs are distributed over 5 clusters. The number of TECs per cluster ranges from 8 to 10 turbines. The clustering is carried out in a mixed chain/star configuration since this type of connection offers the best compromise between the total length and cross-sections of the MV cables in the total investment cost. The TECs are connected by MV cables with a cross-section of $3 \times 185 \mathrm{~mm}^{2}$. The connection between the OSS and the OCP is established by a $3 \times 185 \mathrm{~mm}^{2} \mathrm{HV}$ section cable. The details of the electrical architectures are summarized in Table 3.

Table 3. Optimal electrical architecture.

\begin{tabular}{|c|c|c|}
\hline \multirow{2}{*}{ Quantity } & \multicolumn{2}{|c|}{ Value } \\
\cline { 2 - 3 } & GA & PSO \\
\hline Number of OSSs & 1 & 1 \\
\hline Number of MV feeders per OSS & 5 & 5 \\
\hline clustering & $\begin{array}{c}\text { Mixed } \\
\text { string/star }\end{array}$ & $\begin{array}{c}\text { Mixed } \\
\text { string/star }\end{array}$ \\
\hline Number of TEC per group & $8: 10$ & $8: 10$ \\
\hline Sizes of MV cables (mm $\left.{ }^{2}\right)$ & $3 \times 185$ & $3 \times 185$ \\
\hline Sizes of HV cables (mm $\left.{ }^{2}\right)$ & $3 \times 185$ & $3 \times 185$ \\
\hline Cable length $(\mathrm{km})$ & 25.15 & 25.48 \\
\hline Transformer Power (MVA) & 100 & 100 \\
\hline
\end{tabular}

The convergence of the GA and the PSO algorithm is shown in Fig.7. The GA converges faster than the PSO algorithm (the GA converges to the final solution after about 1500 generations whereas the PSO algorithm converges after about 2000 generations), and towards a slightly better results $\left(\mathrm{F}_{\mathrm{ECT}}=34.39\right.$ using the $\mathrm{GA}$ versus 34.62 using the PSO algorithm). This small difference is due to the total length of cable employed in the two electrical architectures. Indeed, the PSO algorithm provides a connection topology with a higher cable length $(25.45 \mathrm{~km})$ than the GA $(25.15 \mathrm{~km})$. The ECT costs for the two configurations are detailed in Table 4 .

Table 4. Cost of the optimal electrical connection Topology.

\begin{tabular}{|c|c|c|}
\hline \multirow{2}{*}{ Quantity } & \multicolumn{2}{|c|}{ Cost (M€) } \\
\cline { 2 - 3 } & GA & PSO \\
\hline MV ; HV cables & $2.34 ; 8.41$ & $2.50 ; 8.41$ \\
\hline Setup transformer & 1.402 & 1.402 \\
\hline MV/HV transformer & 0.798 & 0.798 \\
\hline MV; HV protection cells & $0.215 ; 0.106$ & $0.215 ; 0.106$ \\
\hline OSS platform & 9 & 9 \\
\hline $\begin{array}{c}\text { Reactive energy } \\
\text { compensation equipment }\end{array}$ & 0.532 & 0.532 \\
\hline Initial investment & 22.81 & 22.96 \\
\hline Investment & 34.26 & 34.62 \\
\hline Cost of losses & 0.134 & 0.138 \\
\hline Fitness of FECT & 34.39 & 34.62 \\
\hline
\end{tabular}


(a)

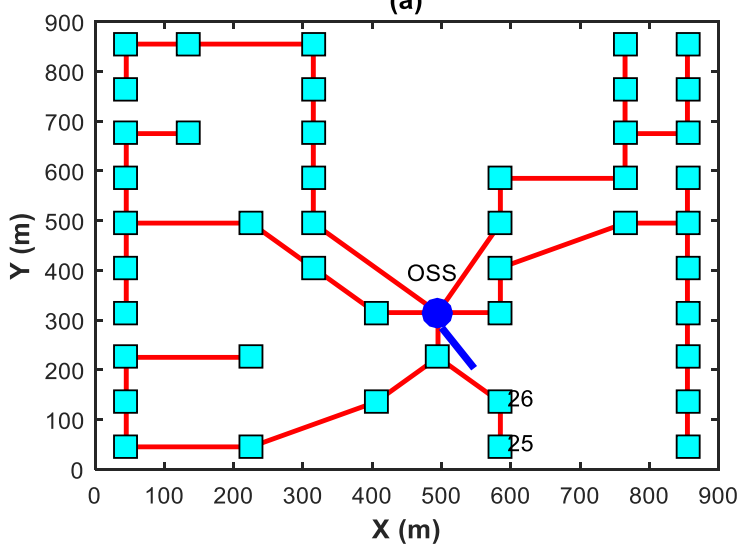

(b)

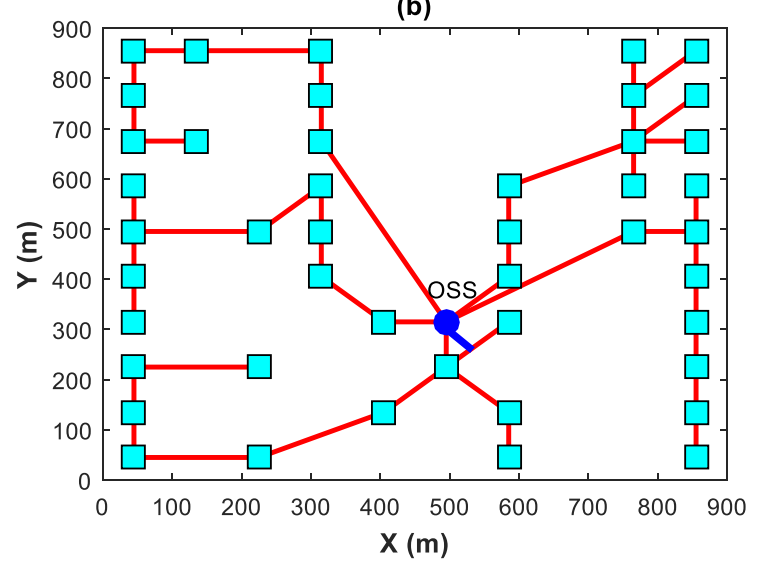

Fig. 6. Optimal ETC. (a) Using a GA (b) Using a PSO.

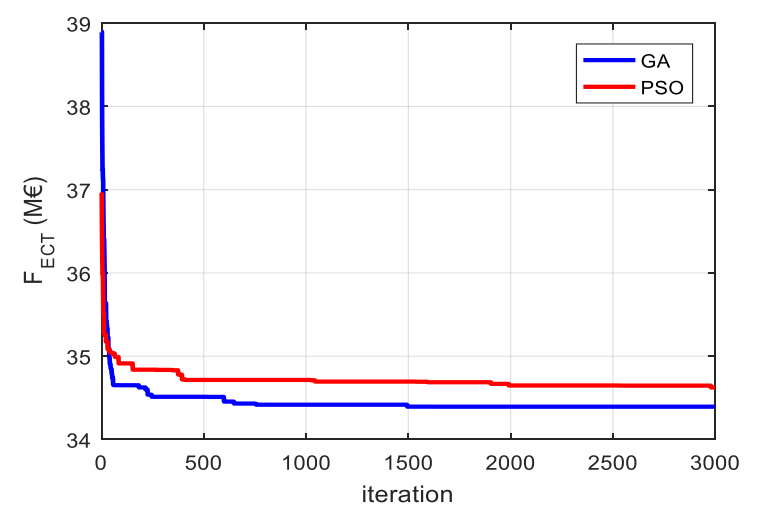

Fig. 7. Convergence of the objective function.

\section{Conclusions}

The economic performance of tidal farms is highly dependent on the design of the electrical connection topology and the sizing of its components. This study has presented an optimization approach based on a genetic algorithm to find the best connection topology of the tidal farm network, containing 45 tidal energy converters located in the Fromveur Strait (France). The approach took into account the energy loss in the submarine cables and a large panel of variables such as the number of tidal energy converters clusters connected to each offshore substation, the number of tidal energy converters in each cluster, the cross-section of MV and
HV cables, the connection design for each cluster of turbines and the number and locations of OSSs.

The results show that it is better to connect the tidal turbines in a mixed chain/star clustering with a central positioning of the offshore substation. This result was confirmed by using a PSO algorithm. The optimal architectures generated by the two algorithms were very similar. The GA converged to a slightly better result than those obtained using the PSO.

In future work, the method presented will be integrated into an optimization tool containing several optimization modules such as the optimization of the positioning of the tidal energy converters within the array.

\section{REFERENCES}

1. S. E. B. Elghali, M. E. H. Benbouzid, and J. F. Charpentier, in 2007 IEEE Int. Electr. Mach. Drives Conf. (IEEE, Antalya, 2007), pp. 1407-1412

2. P. Hou, G. Yang, W. Hu, C. Chen, M. Soltani, and Z. Chen, in 2018 IEEE Congr. Evol. Comput. CEC (IEEE, Rio de Janeiro, 2018), pp. 1-8

3. Chen Ning, Huang Ling-Ling, Zhang Hongyue, and Fu Yang, in Int. Conf. Sustain. Power Gener. Supply SUPERGEN 2012 (Institution of Engineering and Technology, Hangzhou, China, 2012), pp. 67-67

4. M. Zhao, Z. Chen, and F. Blaabjerg, IET Renew. Power Gener. 3, 205 (2009)

5. P. Hou, W. Hu, and Z. Chen, IET Renew. Power Gener. 10, 694 (2016)

6. Z. Chen, C. Chen, W. Hu, and P. Hou, IET Renew. Power Gener. 10, 175 (2016)

7. J. S. González, Á. G. G. Rodríguez, J. C. Mora, M. Burgos Payán, and J. R. Santos, Renew. Energy 36, 1973 (2011)

8. O. Dahmani, S. Bourguet, M. Machmoum, P. Guerin, P. Rhein, and L. Josse, IEEE Syst. J. 9, 1519 (2015)

9. Z. Ren, Y. Wang, H. Li, X. Liu, Y. Wen, and W. Li, IEEE Trans. Power Syst. 34, 292 (2019)

10. Z. Ren, H. Li, Y. Liu, Y. Xu, L. Jin, W. Li, and W. Wang, IEEE Access 7, 71585 (2019)

11. O. Dahmani, S. Bourguet, P. Guerin, M. Machmoum, P. Rhein, and L. Josse, in 2013 IEEE Grenoble Conf. (IEEE, Grenoble, France, 2013), pp. $1-6$

12. S. Lundberg, Performance Comparison of Wind Park Configurations (School of Electrical Engineering. Chalmers University of Technology, Sweden, 2003), p. 214

13. F. M. Gonzalez-Longatt, P. Wall, P. Regulski, and V. Terzija, IEEE Syst. J. 6, 164 (2012)

14. M. Nandigam and S. K. Dhali, in 2008 Int. Symp. Power Electron. Electr. Drives Autom. Motion (IEEE, Ischia, Italy, 2008), pp. 1470-1474

15. M. Zubiaga, G. Abad, J. A. Barrena, S. Aurtenetxea, and A. Cárcar, 10 (n.d.)

16. R. Eberhart and J. Kennedy, in MHS95 Proc. Sixth Int. Symp. Micro Mach. Hum. Sci. (IEEE, Nagoya, Japan, 1995), pp. 39-43 
17. Y. Shi and R. Eberhart, in 1998 IEEE Int. Conf. Evol. Comput. Proc. IEEE World Congr. Comput.
Intell. Cat No98TH8360 (IEEE, Anchorage, AK, USA, 1998), pp. 69-73 